\title{
Minimally invasive glaucoma surgery. A practical guide. (2016) Editors: B. A. Francis, S R Sarkisian Jr., J C Tan. 236 pp. ISBN 978-1-62,623-156-6 e-ISBN 978-1-62,623-155-9 Thieme Publishers New York/Stuttgart
}

\author{
Cecilia Fenerty ${ }^{1}$
}

Received: 13 April 2017 / Accepted: 24 April 2017 / Published online: 16 May 2017

(C) Springer-Verlag Berlin Heidelberg 2017

This excellent book is indeed a very practical guide to the newer minimally invasive glaucoma surgical (MIGS) techniques and will appeal to both glaucoma specialists in training and general ophthalmologists. Currently the only book of its kind, the text goes beyond the focus of the practicalities of these surgical techniques, and discusses the basic sciences of anatomy and physiology pertaining to aqueous production and drainage which underpin the advances in these new technologies.

The hardback book provides 40 chapters over 199 pages written by 76 authors and experts in the field. With so many authors contributing to the book, it is important to maintain consistency in style and content for each chapter, and this is achieved by having a structured approach to each subject.

Section One of the book covers the relevant anatomy and physiology of the eye under the subheadings of trabecular outflow, uveoscleral outflow, aqueous production and subconjunctival filtration. Following a description of the structure and function of each, further chapters follow considering the effects of glaucoma treatment on these anatomical and physiological aspects of the eye.

Section two divides the MIGS procedures under the subheadings of trabecular outflow procedures, uveoscleral outflow procedures, aqueous humour reduction procedures and transconunctival filtration procedures. Again, a consistent structured approach is found in each chapter; a brief clinical case is presented to illustrate the application of each surgical procedure, followed by a proposed rationale for the use of this technology, a discussion regarding appropriate patient selection and a detailed description of the surgical technique itself, including potential complications, postoperative management and overview of the safety and efficacy of the procedure, referencing current literature.

The text is well illustrated throughout with the aid of photographs, clinical imaging techniques and high quality colour illustrations. The book is also supported by 37 short video clips to demonstrate the surgical techniques, however the quality of these is variable with regard to the resolution of the images, centration and the provision of auditory narration or subtext.

This book is a valuable resource for anyone wishing to undertake training in any of the current minimally invasive glaucoma procedures, however its strength is that it is more than just a surgical instruction manual. Inevitably, some of the MIGS techniques described as available may fall out of favour and newer methods may become available. Nevertheless, the detailed description of the anatomy and physiology of aqueous production, including the new knowledge gained through the application of new technologies, as well as the coverage of the advantages, limitations, pitfalls and rationale for MIGS techniques, will ensure this book remains relevant to those managing glaucoma regardless of the changing surgical fashions over time.
Cecilia Fenerty

cecilia.fenerty@cmft.nhs.uk

1 Manchester Royal Eye Hospital, Oxford Road, Manchester M13 9WH, UK 